\title{
Retraction
}

\section{The Stroop effect and single letter coloring: What replicates and what doesn't?}

\author{
DEREK BESNER and JENNIFER A. STOLZ \\ University of Waterloo, Waterloo, Ontario, Canada
}

\section{A (Replicated) Phenomenon}

There are many variants of Stroop's (1935) original paradigm. In one variant, Besner, Stolz, and Boutilier (1997) displayed a color word at fixation. All letters were colored on half the trials; on the remaining trials, only one letter selected at random was colored. The Stroop effect (incongruent minus congruent RT) was reduced when only a single letter was colored, in comparison with when all the letters were colored. Besner and colleagues took this interaction as evidence that any account in which the irrelevant word is always processed (strong automaticity) is problematic. Independent replications and extensions have been reported by Brown, Joneleit, Robinson, and Brown (in press).

\section{Spatially Separating the Color Carrier From the Color Word}

In another variant, Besner and Stolz (1999) displayed a neutral word at fixation, in which all the letters were colored on half the trials. On the remaining trials, only a single letter selected at random was colored. A color word (in white) appeared above or below the display on any one trial (Experiments 1 and 2). Besner and Stolz reported an interaction in which there was no Stroop effect (difference between incongruent and congruent RT) when only a single letter was colored, but a Stroop effect when all letters were colored. In Experiment 3, the material at fixation consisted of a geometric form, and either all its elements were colored, or only a single element was colored. Here, both conditions produced a Stroop effect. It was argued that whether a single element or all elements were colored, together with the nature of the material at fixation, jointly determined whether a Stroop effect was observed. Unfortunately, despite repeated attempts, not all of these results replicate; a series of seemingly unrelated programming errors are the apparent culprit. What does replicate is (1) the observation of a null Stroop effect when only a single letter is colored in the neutral word at fixation, and (2) the presence of a Stroop effect when either all elements are colored or only a single element is colored and the material at fixation does not consist of a word, but is instead "nonlinguistic" (e.g., characters from the top of the keyboard such as “\#”, “*”, “!”, “\%”). What does not replicate, surprisingly, is the presence of a Stroop effect when a neutral word is presented at fixation and all the letters are colored. Instead, across a series of experiments, there was never a significant Stroop effect in this condition. Some of the conclusions originally drawn by Besner and Stolz still hold (i.e., the principle of domain specific processing appears important), but others need modification (within domain capacity limitations appear to play a major role). Any adequate account of "the" Stroop effect must explain both its presence and absences.

\section{REFERENCES}

Besner, D. \& Stolz, J. A. (1999). Unconsciously controlled processing: The Stroop effect reconsidered. Psychonomic Bulletin \& Review, 6, 449-455.

Besner, D., Stolz, J. A., \& Boutilier, C. (1997). The Stroop effect and the myth of automaticity. Psychonomic Bulletin \& Review, 4, 221225.

Brown, T. L., Joneleit, K, Robinson, C. S., \& Brown, C. R. (in press). Automaticity in reading and the Stroop task: Testing the limits of involuntary word processing. American Journal of Psychology.

Stroop, J. R. (1935). Studies of interference in serial verbal reactions. Journal of Experimental Psychology, 18, 643-661.

(Manuscript received December 5, 2001; accepted for publication December 5, 2001.) 